\title{
AN OSCILLATION CRITERION FOR SECOND-ORDER LINEAR DIFFERENTIAL EQUATIONS
}

\section{W. J. COLES}

1. Introduction. The well-known Leighton-Wintner [2], [3] oscillation theorem for

$$
y^{\prime \prime}+p(x) y=0 \quad p(x) \text { continuous on }[0, \infty)
$$

is

Theorem (i). Equation (1) is oscillatory on $[0, \infty)$ if

$$
\int^{\infty} p=+\infty \text {. }
$$

The case

$$
\liminf _{x \rightarrow \infty} \int^{x} p<+\infty
$$

remains of interest and can produce either oscillatory or nonoscillatory behavior.

Let

$$
P(x)=\frac{1}{x} \int_{0}^{x} \int_{0}^{t} p(s) d s d t .
$$

Hartman [1] has proved that nonoscillation of (1) implies that either $P(x)$ tends to a finite limit or else that $\lim \inf _{x \rightarrow \infty} P(x)=-\infty$, so that one has:

Theorem (ii). $-\infty<\lim \inf _{x \rightarrow \infty} P(x)<\lim \sup _{x \rightarrow \infty} P(x)$ implies oscillation.

Theorem (iii). $\lim _{x \rightarrow \infty} P(x)=+\infty$ implies oscillation.

Since (2) implies the hypothesis of (iii), Theorem (iii) implies Theorem (i).

The above theorems do not apply if $P(x)$ tends to a finite limit or if $\lim \inf _{x \rightarrow \infty} P(x)=-\infty$; e.g., they give no information about such coefficients as $p(x)=(x \cos x-\sin x) / x^{2}$ or $p(x)=x^{2} \sin x$. The purpose of this note is to derive oscillation criteria for certain classes of such coefficients.

Received by the editors March 20, 1967. 
2. Weighted averages. The idea is that additional information about oscillation of (1) may be gained by considering weighted averages of $\int^{x} p$. Let $f$ be a nonnegative, locally integrable function such that $\int_{0}^{x} f \not \equiv 0$; then there is an $a>0$ such that

$$
A(x)=A(f ; p)(x)=\int_{0}^{x} f(t) \int_{0}^{t} p(s) d s d t / \int_{0}^{x} f(t) d t
$$

exists on $[a, \infty)$.

THEOREM 1. If there exists a nonnegative, locally integrable function $f$ satisfying

$$
\begin{aligned}
\int_{a}^{\infty}\left\{f(t)\left(\int_{0}^{t} f(s) d s\right)^{k} / \int_{0}^{t} f^{2}(s) d s\right\} d t=+\infty \\
\text { for some } k, 0 \leqq k<1 \text {, and for } a>0
\end{aligned}
$$

and

$$
\lim _{x \rightarrow \infty} A(x)=+\infty
$$

then (1) is oscillatory.

Proof. The proof uses ideas of Hartman. We give a proof for $f$ continuous; the proof is easily modified for $f$ locally integrable. Also, if convenient, we will change the lower limits of the integrals in (5) and (6), since the asymptotic behavior as $x \rightarrow \infty$ is not changed thereby.

Suppose that (1) is nonoscillatory; then, for large enough $a$, a solution of the Riccati equation

$$
z^{\prime}+z^{2}+p(x)=0
$$

exists on $[a, \infty)$. Integration, multiplication by $f$, and in tegration give

$$
\int_{a}^{x} f(t) z(t) d t+\int_{a}^{x} f(t) \int_{a}^{t} z^{2}(s) d s d t=(z(a)-A(x)) \int_{a}^{x} f(t) d t .
$$

By hypothesis, the right-hand side tends to $-\infty$; hence, for large enough $x$,

$$
\int_{a}^{x} f(t) z(t) d t+\int_{a}^{x} f(t) \int_{a}^{t} z^{2}(s) d s d t<0
$$

so that 


$$
\begin{aligned}
\left(\int_{a}^{x} f(t) \int_{a}^{t} z^{2}(s) d s d t\right)^{2} & \leqq\left(\int_{a}^{x} f(t) z(t) d t\right)^{2} \\
& \leqq \int_{a}^{x} f^{2}(t) d t \cdot \int_{a}^{x} z^{2}(t) d t .
\end{aligned}
$$

Let $R(x)=\int_{a}^{x} f(t) \int_{a}^{t} z^{2}(s) d s d t$. Since, for $x \geqq b>a, R(x) \geqq \int_{b}^{x} f(t) d t \cdot \int_{a}^{b} z^{2}(t) d t$, we have from (10) that

$$
f(x)\left(\int_{b}^{x} f(t) d t\right)^{k}\left(\int_{a}^{b} z^{2}(t) d t\right)^{k} / \int_{a}^{x} f^{2}(t) d t \leqq R^{k-2}(x) R^{\prime}(x) .
$$

For $b>a$, integration now gives

$$
\begin{aligned}
\int_{b}^{x}\left\{f(t)\left(\int_{b}^{t} f(s) d s\right)^{k}\right. & \left./ \int_{a}^{t} f^{2}(s) d s\right\} \\
& \leqq \frac{1}{h}\left(\frac{1}{R^{h}(b)}-\frac{1}{R^{h}(x)}\right)<\frac{1}{h R^{h}(b)} \quad(h=1-k)
\end{aligned}
$$

contradicting (6).

Note that (6) implies that

$$
\int^{\infty} f(t) d t=+\infty
$$

a reasonable condition for a weight function. Conversely, if $f$ is bounded then (11) implies (6) for any $k$ such that $0 \leqq k<1$.

If (6) holds for some $k$ on $[0,1)$, it holds for $k=0$; but one advantage in stating the theorem for $k>0$ is that weight functions $x^{\alpha}(\alpha>0)$ are permitted.

The following corollary says roughly that if $\int_{0}^{x} p$ is large enough on a large enough set, then (1) is oscillatory regardless of the behavior of $\int_{0}^{x} p$ on the rest of the half line.

Corollary 1. Let $S(x)=\left\{t \mid 0 \leqq t \leqq x\right.$ and $\left.\int_{0}^{t} p>0\right\}$, and let $m(S(x))$ be the measure of $S(x)$. If $m(S(x)) \rightarrow \infty$ as $x \rightarrow \infty$ and if

$$
\frac{1}{m(S(x))} \int_{S(x)} \int^{t} p(s) d s d t \rightarrow \infty
$$

as $x \rightarrow \infty$, then (1) is oscillatory.

Proof. Take $f(x)$ to be 1 if $\int_{0}^{x} p>0$ and 0 otherwise, let $k=0$, and apply Theorem 1 . 
Examples. If $p(x)=x \sin x$, Theorems (i) and (iii) fail, but Theorem (ii) and Corollary 1 apply.

If $p(x)=x^{2} \sin x$, Theorems (i), (ii), and (iii) fail, but Corollary 1 applies.

Even if no suitable weight function exists for Theorem 1, (1) may oscillate, as the following theorem implies.

THEOREM 2. If $P(x)$ does not approach a finite limit as $x \rightarrow \infty$ and if there is a nonnegative, locally integrable function $f$ satisfying (6) and

$$
\liminf _{x \rightarrow \infty} A(x)>-\infty,
$$

then (1) is oscillatory.

The interesting way for $P(x)$ to fail to have a limit is

$$
\lim _{x \rightarrow \infty} \inf P(x)=-\infty \text {; }
$$

this is the only case not covered by Theorems (ii) and (iii).

Proof of Theorem 2. First we remark that since $\int \infty f=+\infty$ if $g(x)$ is nondecreasing in $x$, we have:

(a) $\int x f(t) g(t) d t / \int x f(t) d t$ is nondecreasing in $x$;

(b) if $\int x f(t) g(t) d t / \int x f(t) d t$ is bounded on $[a, \infty)$, so is $g(x)$.

Suppose that (1) is nonoscillatory. Via the Riccati equation we have (by (12))

$$
\begin{aligned}
{\left[\int_{a}^{x} f(t) z(t) d t\right.} & \left.+\int_{a}^{x} f(t) \int_{a}^{t} z^{2}(s) d s d t\right] / \int_{a}^{x} f(t) d t \\
& =z(a)-A(x) \leqq K \quad(K \text { constant }) \text { on }[b, \infty), b>a .
\end{aligned}
$$

We claim that $\int_{a}^{x} f(t) \int_{a}^{t} z^{2}(s) d s d t / \int_{a}^{x} f(t) d t$ is bounded on $[b, \infty)$. If not, by (a), it tends to $+\infty$; and so, for large $x$,

$$
\begin{aligned}
\int_{a}^{x} f(t) z(t) d t & +\frac{1}{2} \int_{a}^{x} f(t) \int_{a}^{t} z^{2}(s) d s d t \\
\leqq & \left(K-\left(\int_{a}^{x} f(t) \int_{a}^{t} z^{2}(s) d s d t / 2 \int_{a}^{x} f(t) d t\right)\right) \int_{a}^{x} f(t) d t<0 .
\end{aligned}
$$

Now one proceeds as in the proof of Theorem 1 to contradict (6).

So, by (b), $\int^{\infty} z^{2}<\infty$. But Hartman [1] has shown that if (1) is nonoscillatory, then $\int^{\infty} z^{2}<\infty$ if and only if $P(x)$ has a finite limit as $x \rightarrow \infty$. This contradiction completes the proof.

ExAmple. Let $p(x)$ be such that $\int_{0}^{x} p$ is 0 on $[2 n, 2 n+1]$ $(n=0,1, \cdots)$ but is sufficiently negative on $(2 n+1,2 n+2)$ 
$(n=0,1, \cdots)$ to produce $\lim \inf _{x \rightarrow \infty} P(x)=-\infty$. Let $f(x)$ be 1 on $[2 n, 2 n+1](n=0,1, \cdots)$ and 0 elsewhere so that $A(x) \equiv 0$ on $(0, \infty)$. Theorems (i), (ii), (iii), and 1 do not apply, but Theorem 2 does.

3. The general selfadjoint case. Corresponding to Theorems 1 and 2 are Theorems $1^{\circ}$ and $2^{\circ}$ for the equation

$\left(1^{\circ}\right)\left(r(x) y^{\prime}\right)^{\prime}+p(x) y=0(r(x)>0 ; r(x)$ and $p(x)$ continuous on $[0, \infty))$.

Equation (6) becomes

$$
\int_{0}^{\infty}\left\{f(t)\left(\int_{0}^{t} f(s) d s\right)^{k} / \int_{0}^{t} r(s) f^{2}(s) d s\right\} d t=+\infty .
$$

The proofs parallel those of Theorems 1 and 2 so are omitted here. The result of Hartman, needed in the proof of Theorem 2, holds for $\left(1^{\circ}\right)$ with $\int{ }^{\infty} z^{2}$ replaced by $\int{ }^{\infty} z^{2} / r$.

The Leighton-Wintner theorem for $\left(1^{\circ}\right)$, namely that $\left(1^{\circ}\right)$ is oscillatory if $\int^{\infty} p=\int^{\infty} 1 / r=+\infty$, follows from Theorem $1^{\circ}$ on taking $f=1 / r$ and $k=0$. On the other hand, if $\left(6^{\circ}\right)$ holds for some $f$, then $\int^{\infty} 1 / r=+\infty$; and (7) implies that $\lim \sup _{x \rightarrow \infty} \int{ }^{x} p=+\infty$. Thus Theorem $1^{\circ}$ (and Theorem 1) are interesting only in case $\int^{\infty}(1 / r)=+\infty$, $\lim \inf _{x \rightarrow \infty} \int x p<\lim \sup _{x \rightarrow \infty} \int x p=+\infty$.

As with Theorem 2, the interesting case of Theorem $2^{\circ}$ is $\lim \inf _{x \rightarrow \infty} P(x)=-\infty$.

\section{REFERENCES}

1. P. Hartman, On nonoscillatory linear differential equations of second order, Amer. J. Math. 74 (1952), 389-400.

2. W. Leighton, On self-adjoint differential equations of second order, J. London Math. Soc. 27 (1952), 37-47.

3. A. Wintner, A criterion of oscillatory stability, Quart. Appl. Math. 7 (1949), 115-117.

UNIVERSITY OF UTAH 